\title{
Gordon syndrome in an 11-year-old boy: long-term follow-up
}

\author{
Andrzej Badeński', Marta Badeńska', Omar Bjanid', Justyna Czubilińska-Łada1', \\ Aleksandra Gliwińska', Anna Bogdał², Olga Kupczyk³, Maria Szczepańska1 \\ ${ }^{1}$ Chair and Department of Paediatrics, Faculty of Medical Sciences in Zabrze, Medical University of Silesia in Katowice, Poland \\ ${ }^{2}$ Department of Paediatrics, District Hospital in Zawiercie, Poland \\ ${ }^{3}$ Department of Paediatric Nephrology with Dialysis Division for Children, Independent Public Clinical Hospital No. 1, Zabrze, \\ Poland
}

\section{ABSTRACT}

Gordon syndrome, or type 2 pseudohypoaldosteronism, is a rare familial occurring hypertension, in most cases inherited in an autosomal dominant manner. It is characterised by coexisting hyperkalaemia, which is not found in other monogenic forms of hypertension. In addition, in this syndrome aldosterone levels are usually low, and renin activity is reduced under normal salt loading. Renal function, assessed on the basis of glomerular filtration rate, is normal, but metabolic acidosis - type IV renal tubular acidosis - may occur. The authors would like to present the case of an 11-year-old boy in whom the above-described symptoms of Gordon syndrome were observed; clinically effective thiazide diuretic therapy, and subsequent genetic analysis confirmed the diagnosis.

\section{KEY WORDS:}

hyperkalaemia, renal tubular acidosis, pseudohypoaldosteronism type 2 , monogenic arterial hypertension, rare genetic disease.

\section{INTRODUCTION}

Arterial hypertension, according to recent studies, is present in 3-5\% of children and adolescents. Data from the OLAF and OLA studies revealed that the prevalence of this disease among the youngest age group of 3- to 6 -year-olds reached $6.9 \%$ of the corresponding population; subsequently, among children aged 6 to 10 years it was $7.7 \%$, and $6.2 \%$ among adolescents [1]. In the paediatric population, secondary hypertension still dominates, but with the obesity epidemic, which also affects the population of Polish children, primary hypertension has become the leading group in adolescents. In recent decades, genetic hypertension, which is resistant to traditional antihypertensive treatment, has been identified and described as monogenic hypertension (Table 1).
The first report of a patient with hypertension and severe hyperkalaemia appeared in the 1960s [2]. Richard Gordon presented the second case in 1964 [3], and he described it as a hereditary phenotype in several Australian families he had observed. That research led to the idea of a genetic basis of the disease. Gordon syndrome (GS) is mainly inherited as an autosomal dominant trait. To date, it has not been established whether the clinical features differ between different GS genotypes.

TABLE 1. Types of hypertension

\begin{tabular}{|l|c|}
\hline Hypertension & Definition \\
\hline Primary (idiopathic) & The cause is unknown \\
\hline Secondary & Accompanying a specific disease \\
\hline Monogenic & Associated with single gene mutation \\
\hline
\end{tabular}

\section{ADDRESS FOR CORRESPONDENCE:}

Maria Szczepańska, Chair and Department of Paediatrics, Faculty of Medical Sciences in Zabrze, Medical University of Silesia in Katowice, 13/15 3 Maja St., 41-800 Zabrze, Poland, ORCID: 0000-0002-6772-1983, e-mail: szczep57@poczta.onet.pl 
Patients with this phenotype present normal renal function, as assessed by glomerular filtration rate, but may manifest metabolic acidosis - as renal tubular acidosis type IV. Their plasma renin activity is reduced under normal salt loading. However, aldosterone levels are usually low due to severe hyperkalaemia $(8-9 \mathrm{mmol} / \mathrm{l})$. In fact, either salt restriction ( $\leq 20 \mathrm{mmol} /$ day) or low doses of thiazide diuretics are very effective in normalising blood pressure values and electrolyte disorders in patients with GS $[4,5]$. Hyperkalaemia is a major distinguishing factor because all other monogenic syndromes that increase blood pressure do not cause changes in plasma potassium levels or lead to obvious hypokalaemia [6]. Metabolic abnormalities, hyperkalaemia, and hyperchloraemic metabolic acidosis usually precede hypertension, and hypertension often only manifests itself in adulthood [7,8]. What is more, hypercalciuria occurs in most patients with GS $[8,9]$.

There are descriptions of a condition considered to be an early syndrome of GS: Spitzer-Weinstein syndrome occurs in childhood and consists of hyperkalaemia, metabolic acidosis, and growth failure, but not hypertension [10-13].

In this manuscript we will describe the case of Gordon's syndrome as the example of monogenic hypertension with a long-term follow-up, to present the course of the disease and the administered treatment.

\section{CASE REPORT}

An 11-year-old boy suffering from pseudohypoaldosteronism type 2 (PHA 2), under constant nephrological control since early childhood.

TABLE 2. Height standard deviation score

\begin{tabular}{|l|c|}
\hline Age (years) & Height standard deviation score \\
\hline 2.5 & -0.92 \\
\hline 3 & -1.43 \\
\hline 3.5 & -1.0 \\
\hline 4 & -1.13 \\
\hline 4.5 & -1.2 \\
\hline 5 & -1.47 \\
\hline 5.5 & -1.43 \\
\hline 6 & -1.87 \\
\hline 6.5 & -1.44 \\
\hline 7 & -1.65 \\
\hline 7.5 & -1.56 \\
\hline 8 & -1.52 \\
\hline 8.5 & -1.3 \\
\hline 9 & -1.3 \\
\hline 9.5 & -1.39 \\
\hline 10 & -1.17 \\
\hline 10.5 & -1.2 \\
\hline
\end{tabular}

History of pregnancy, neonatal period, and development were burdened. During pregnancy, the mother was hospitalised due to abdominal pain and urinary tract infection. The neonate was born entirely at $34^{\text {th }}$ week of gestational age, by caesarean section due to premature drainage of amniotic fluid, after the fourth pregnancy and the fourth delivery of the mother, with a birth weight of $2000 \mathrm{~g}$. He obtained an Apgar score of 4/7/7/8 points in consecutive evaluations. Postnatal functioning was complicated by immaturity, congenital pneumonia, and perinatal asphyxia.

Family history of chronic diseases was also burdened - the mother suffered from arterial hypertension and additionally the maternal grandmother had arterial hypertension with hyperkalaemia, but no further diagnosis or genetic tests were performed.

At the age of two years, the boy was admitted to the paediatric nephrology department presenting mild hyperkalaemia, normal sodium and aldosterone levels, reduced plasma renin activity, and elevated blood pressure values. During the examination, apart from body stature below the third percentile (Table 2) and borderline blood pressure measurements (approximately $90^{\text {th }}$ percentile during sleep), the boy was in good general condition. His physical and mental development seemed normal - he pronounced a few single words.

During the hospitalisation, hyperkalaemia with hyporeninaemia occurred, but aldosterone levels were normal. There was also a periodic normochloraemic metabolic acidosis tendency in blood gas analysis (Table 3). Although the urine $\mathrm{pH}$ was acidic, a highly positive anion gap was found in the urine.

The ECG was within the normal age range. Ultrasound examination of the heart showed left ventricular thickness at the upper limit of normal, the remaining structures of the heart were unaffected. Ultrasound examination of the abdomen did not reveal any pathologies. The thyroid gland was normal in ultrasound.

The boy was prescribed a low-salt diet and pharmacotherapy. Hydrochlorothiazide in a daily dose of $4.125 \mathrm{mg}$ and amlodipine in a daily dose of $8.75 \mathrm{mg}$ were applied, which successfully improved the patient's condition and normalised his blood pressure.

The presented clinical symptoms and laboratory tests strongly corresponded to the characteristics of Gordon syndrome. Consequently, genetic tests were performed in order to confirm the diagnosis. The outcome of the research revealed the mutation of the with-no-lysine $(\mathrm{K})$ protein 1 (WNK1).

The patient remained under constant ambulatory observation, which included, among others, blood pressure examinations (Fig. 1A), blood gas analysis, and serum potassium level analysis (Fig. 1B). During the first year of treatment, the dose of hydrochlorothiazide was increased twice, due to insufficient treatment results. In the third year of life, during control tests, severe hyperkalaemia 
TABLE 3. Laboratory tests

\begin{tabular}{|c|c|c|c|c|c|c|}
\hline \multirow[t]{2}{*}{ Parameter } & \multicolumn{5}{|c|}{ Hospitalisations } & \multirow{2}{*}{ Reference ranges } \\
\hline & At age 2 & At age 4 & At age 6 & At age 8 & At age 10 & \\
\hline \multicolumn{7}{|l|}{ Blood gas analysis } \\
\hline pH & 7.39 & 7.399 & 7.423 & 7.393 & 7.442 & $7.35-7.45$ \\
\hline $\mathrm{pCO}_{2}(\mathrm{~mm} \mathrm{Hg})$ & 35.8 & 45.2 & 37.4 & 38.7 & 28.7 & $35-45$ \\
\hline $\mathrm{pO}_{2}(\mathrm{~mm} \mathrm{Hg})$ & 75.9 & 67.9 & 84.7 & 69.5 & 78.2 & $65-100$ \\
\hline $\mathrm{HCO}_{3} \mathrm{act}(\mathrm{mmol} / \mathrm{l})$ & 21.0 & 27.3 & 23.9 & 23.1 & 19.1 & $21-27$ \\
\hline $\mathrm{ctCO}_{2}(\mathrm{mmol} / \mathrm{l})$ & 22.1 & 28.7 & 25.0 & 24.2 & 20 & $22-28$ \\
\hline $\mathrm{BE}(\mathrm{b})(\mathrm{mmol} / \mathrm{l})$ & -3.3 & 2.0 & -0.2 & -1.5 & -3.5 & $-2.5-2.5$ \\
\hline $0_{2}$ SAT $(\%)$ & 95.2 & 93.5 & 96.6 & 93.9 & 96.2 & \\
\hline \multicolumn{7}{|l|}{ Diagnostic tests } \\
\hline $\begin{array}{l}\text { Serum aldosterone } \\
\text { concentration }(\mathrm{pg} / \mathrm{ml})\end{array}$ & 495.6 & - & - & - & - & $140-560$ \\
\hline $\begin{array}{l}\text { Plasma renin activity (ng/ml/h) } \\
\text { (upright posture) }\end{array}$ & 0.6 & - & - & - & - & $5.6-7.4$ \\
\hline Alkaline phosphatase (U/I) & 163.0 & - & 213 & 184 & 232 & $100-230$ \\
\hline Total protein $(\mathrm{g} / \mathrm{l})$ & 59.70 & 62.1 & 72.8 & 72.0 & 70.7 & $60-80$ \\
\hline Serum urea $(\mathrm{mmol} / \mathrm{l})$ & 3.00 & 3.5 & 3.1 & 2.3 & 5.4 & $3.2-7.1$ \\
\hline Serum creatinine $(\mu \mathrm{mol} / \mathrm{l})$ & 36.00 & 41.0 & 46.0 & 49.0 & 50 & $21-53$ \\
\hline $\begin{array}{l}\text { eGFR by new Schwartz formula } \\
\left(\mathrm{ml} / \mathrm{min} / 1.73 \mathrm{~m}^{2}\right)\end{array}$ & 86.2 & 87.27 & 88.89 & 92.3 & 96.38 & \\
\hline Serum magnesium (mmol/l) & 0.85 & 0.84 & 0.77 & 0.73 & 0.76 & $0.7-1.1$ \\
\hline Serum sodium (mmol/l) & 139.5 & 137.3 & 142 & 139 & 141 & $135-148$ \\
\hline Serum phosphate (mmol/l) & 1.88 & 1.5 & 1.33 & 1.28 & 1.31 & $0.81-1.45$ \\
\hline Serum potassium (mmol/l) & 6.29 & 3.9 & 4.53 & 5.03 & 4.51 & $3.50-5.00$ \\
\hline Serum total calcium (mmol/l) & 2.58 & 2.44 & 2.45 & 2.52 & 2.43 & $2.10-2.55$ \\
\hline Serum ionised calcium (mmol/l) & 1.21 & 1.14 & 1.1 & 1.11 & 0.98 & $1.13-1.32$ \\
\hline Chloride (mmol/l) & 103.1 & 99.3 & 104.1 & 103.6 & - & $98-125$ \\
\hline Urine potassium (mmol/l) & 19.70 & 83.96 & 30.3 & 28.12 & 29.41 & $25-100$ \\
\hline Urine sodium (mmol/l) & 98.0 & 110.0 & 107 & 152 & 138 & $80-240$ \\
\hline Urine anion gap (mEq/l) & +50 & - & - & - & - & $0-20$ \\
\hline \multicolumn{7}{|l|}{ Daily urine collection } \\
\hline Creatinine (mg/kg/24 h) & 8.04 & 12.23 & 20.54 & 13.49 & 14.18 & $5-25$ \\
\hline Uric acid (mg/kg/24 h) & 5.46 & 7.88 & 11.75 & 5.62 & 8.27 & $\leq 12$ \\
\hline Urea (mg/kg/24 h) & 129.33 & 112.41 & 282.41 & 183.24 & 281 & $>300$ \\
\hline $\mathrm{Mg}(\mathrm{mg} / \mathrm{kg} / 24 \mathrm{~h})$ & 1.14 & 0.95 & 2.45 & 1.53 & 1.83 & $\leq 2$ \\
\hline$P(\mathrm{mg} / \mathrm{kg} / 24 \mathrm{~h})$ & 5.49 & 6.68 & 8.38 & 7.95 & 5.39 & $15-25$ \\
\hline $\mathrm{Ca}(\mathrm{mg} / \mathrm{kg} / 24 \mathrm{~h})$ & 1.11 & 0.34 & 0.97 & 0.88 & 4.89 & $1-4$ \\
\hline $\mathrm{Na}(\mathrm{mmol} / \mathrm{kg} / 24 \mathrm{~h})$ & 1.77 & 1.15 & 4.57 & 2.87 & 3.15 & $1-4$ \\
\hline $\mathrm{K}(\mathrm{mmol} / \mathrm{kg} / 24 \mathrm{~h})$ & 0.43 & 0.88 & 1.29 & 0.53 & 0.66 & $1-2$ \\
\hline
\end{tabular}




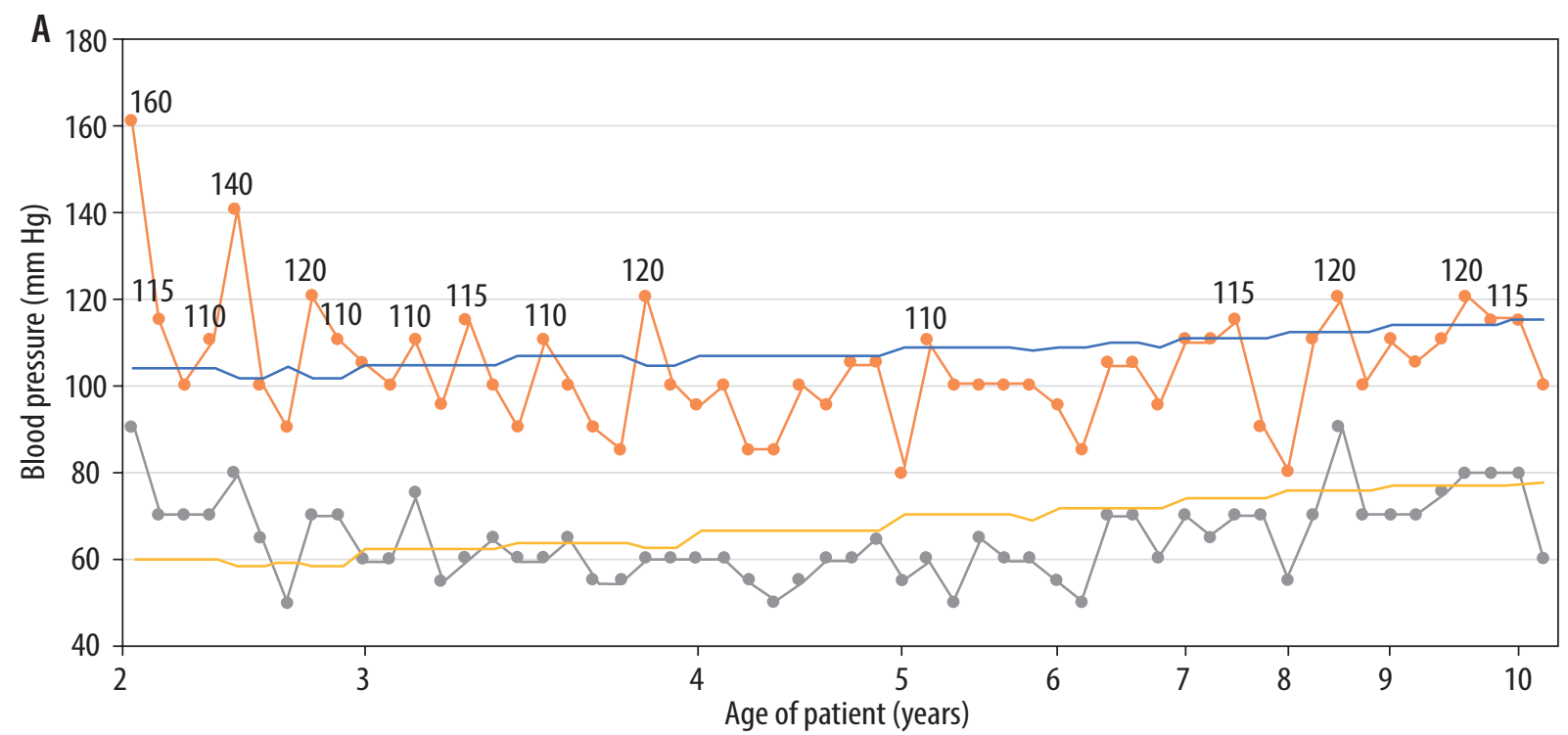

— $95 \mathrm{pc}$ for systolic blood pressure _ $95 \mathrm{pc}$ for diastolic blood pressure

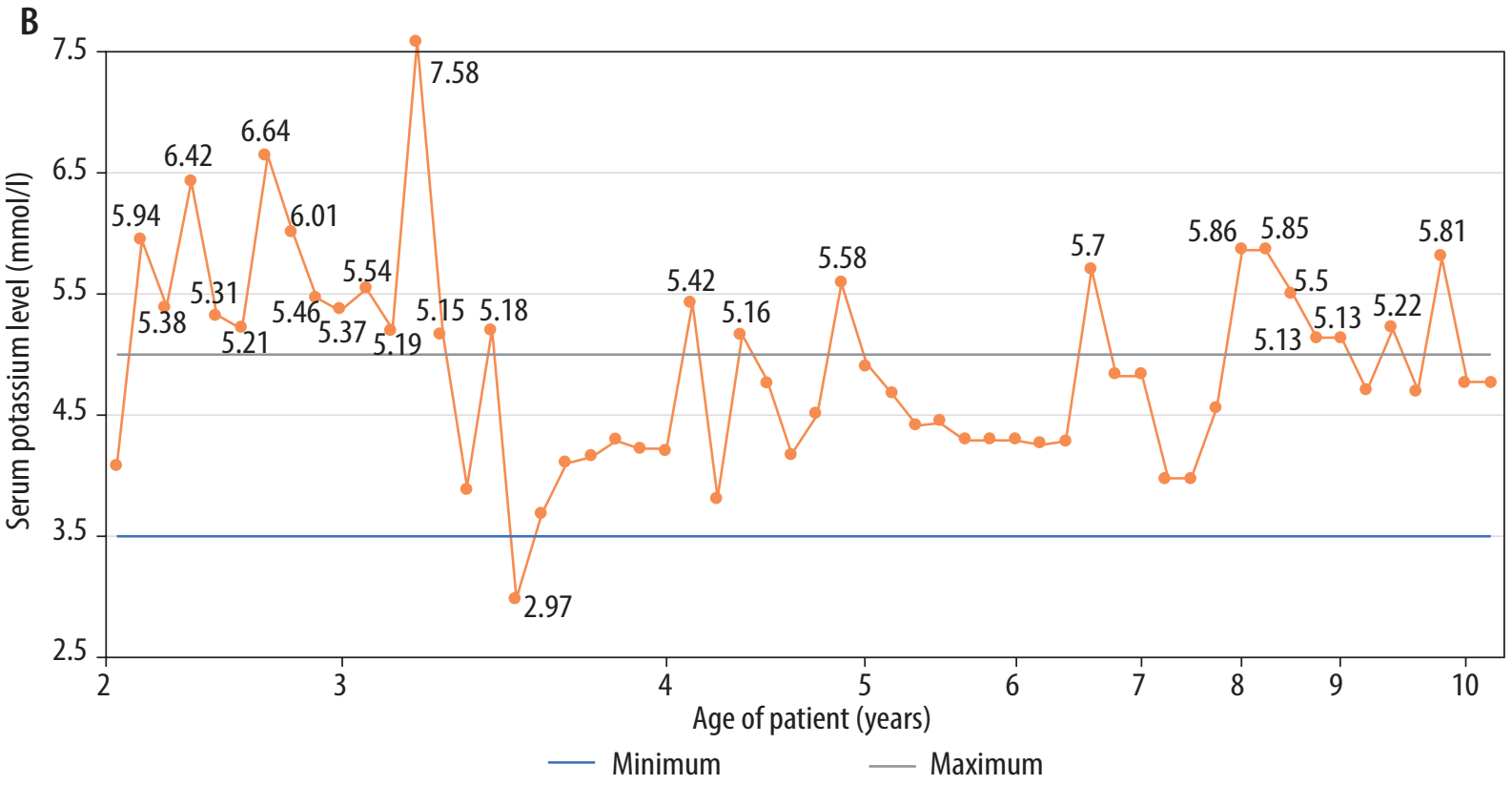

FIGURE 1. A) Blood pressure and B) serum potassium level

occurred - serum potassium level reached $7.58 \mathrm{mmol} / \mathrm{l}$. Therefore, the boy was immediately admitted to the hospital. During this hospitalisation, the dose of hydrochlorothiazide was increased again, with strong clinical response. Consequently, the serum potassium level decreased rapidly, which confirmed the diagnosis of GS by the significant outcome of thiazide administration. The treatment was modified once more, and it reached a total daily dose of $5.125 \mathrm{mg}$. The amlodipine therapy was maintained due to a good clinical response.

At the age of five years the boy was admitted to the hospital for control tests. He was then referred to the psychologist due to his aggressive behaviour. He was observed for oppositional defiant disorder. In the following four years the patient was annually admitted to the hospital for control tests. During each hospitalisation, 24-hour blood pressure monitoring and echocardiogram were performed - the results were within normal limits; therefore, the existing treatment was maintained. When the patient was nine years old, 24-hour blood pressure monitoring showed blood pressure values above the $95^{\text {th }}$ percentile for sex, age, and height. Consequently, the dose of hydrochlorothiazide was increased to total daily dose of $6.25 \mathrm{mg}$.

\section{DISCUSSION}

Mineralocorticoid hormones, produced by the adrenal cortex, play a major role in maintaining fluid and electrolyte balance and providing normal blood pressure range. Their main function is to reabsorb sodium, chloride, and water through the renal tubules. In monogenic 
TABLE 4. Types of Gordon syndrome

\begin{tabular}{|c|c|c|c|}
\hline Disease & Inheritance & Cytogenetic loci & Gene \\
\hline \multirow{5}{*}{$\begin{array}{l}\text { Gordon syndrome } \\
\text { (pseudohypoaldosteronism } \\
\text { type II) }\end{array}$} & Autosomal dominant (type II A) & $1 q 31-1 q 42$ & - \\
\hline & Autosomal dominant (type II B) & $17 q 21.2$ & WNK4 \\
\hline & Autosomal dominant (type II C) & $12 \mathrm{p} 13.33$ & WNK1 \\
\hline & Autosomal dominant or recessive (type II D) & $5 q 31.2$ & KLHL3 \\
\hline & Autosomal dominant (type II E) & $2 q 36.2$ & CUL3 \\
\hline
\end{tabular}

hypertensive disorders, mutations of a single gene lead to a primary increase in the rate of reabsorption of sodium and chloride in the distal nephron or to enhancement of the action of hormones with mineralocorticoid activity. Hypertension develops as a result of increased reabsorption of sodium, chloride, and water, followed by an increase in plasma volume.

The evaluation of the renin-angiotensin-aldosterone system (RAAS) is a crucial part of the initial diagnostic process in children with a suspected monogenic form of arterial hypertension. All monogenic forms of hypertension are characterised by low renin activity. Consequently, normal range of plasma renin activity excludes the diagnosis of mendelian hypertension.

The patient described in this article had high blood pressure values since early childhood. Electrolyte abnormalities, such as metabolic acidosis with hyperkalaemia, were marked in his laboratory tests. These abnormalities are considered to be relevant markers for the first suspicion of GS. It was decided to extend the diagnostic process. Moreover, an aspect worth drawing attention to is the strong clinical response to the hydrochlorothiazide treatment. Thiazides are responsible for the suppression of the sodium-chloride symporter (NCC); therefore, the sodium reabsorption decreased, which resulted in an improvement of the boy's condition and normalisation of the clinical and laboratory outcome. To confirm the diag-

A

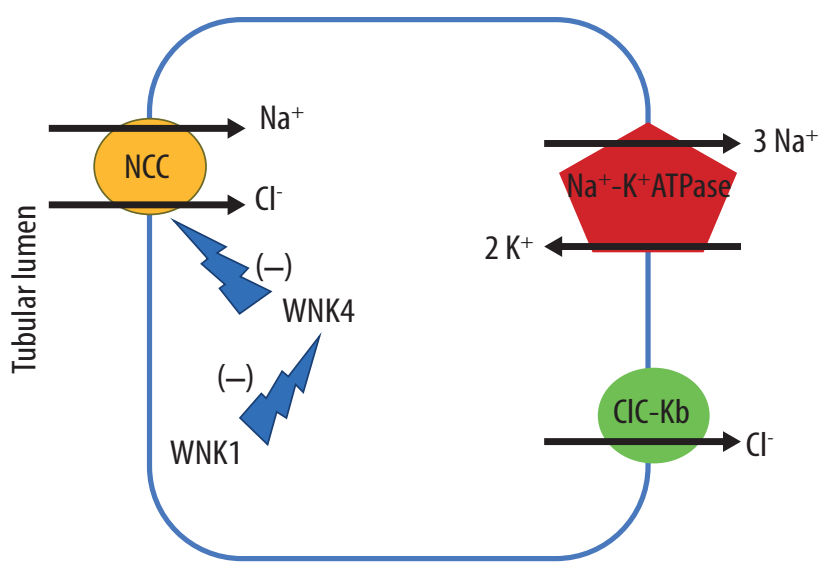

FIGURE 2. A) Distal convoluted tubule, B) collecting duct nosis of GS it is obligatory to perform genetic tests, which reveal the causative mutation. In the presented case, mutation in the WNK 1 gene was observed; therefore, PHA 2 type $\mathrm{C}$ was diagnosed.

According to the literature, PHA II is frequently inherited in an autosomal dominant manner, whereas the minority arise de novo. Therefore, it is recommended that prenatal diagnosis be performed if the pathogenic variant is known in the family, and then the proper treatment be provided to prevent patients from developing secondary complications, such as cardiovascular and renal disease or stroke [14].

\section{PATHOPHYSIOLOGY OF GORDON SYNDROME}

The pathophysiology of PHA type II was firstly reported to be related to the mutation in genes coding for with-no-lysine (K) (WNK) serine-threonine kinase family (WNK1 and WNK4) $[15,16]$. More recently, mutations in Kelch-like 3 (KLHL3) and Cullin 3 (CUL3) were discovered also to cause PHA II $[17,18]$ (Table 4). These abnormalities interfere with the regulation of fluid, sodium, and potassium concentrations at several levels within the nephron.

The clinical manifestation varies depending on which mutation occurs. Most frequently, milder forms of PHA II refer to cases of WNK 1 and WNK 4 muta-

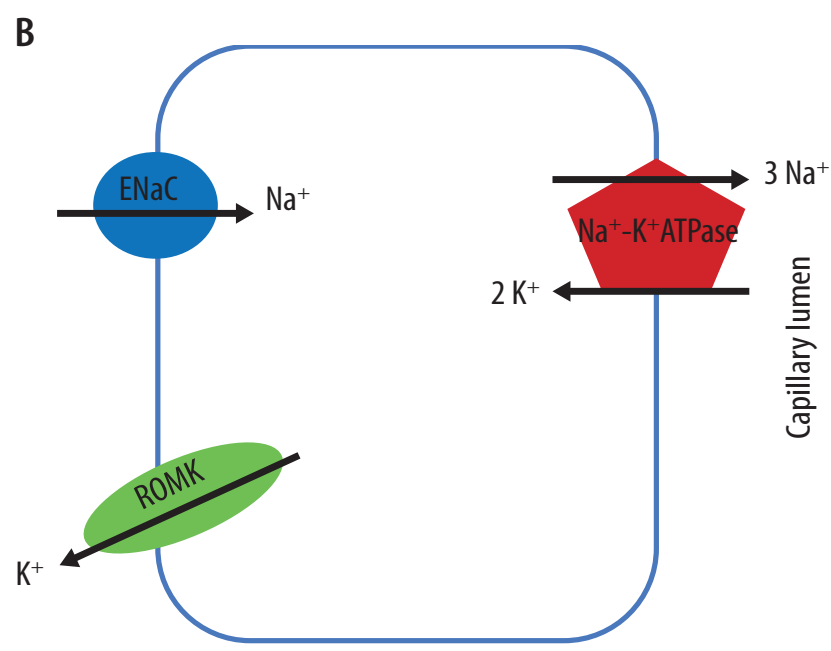

NCC - sodium-chloride symporter, CIC-Kb-basolateral chloride channel, WNK1 - WNK1 kinase, WNK4 - WNK4 kinase, ENaC - epithelial sodium channel, ROMK - renal outer medullary potassium channel 
tions. Patients with a heterozygous CUL3 pathogenic variant are more likely to develop severe hyperkalaemia and metabolic acidosis, hypertension at an early stage, and growth impairment in comparison with individuals with KLHL3, WNK1, or WNK4 alterations [17]. Autosomal recessive inheritance of KLHL3 pathogenic variants usually results in more severe phenotype than does the autosomal dominant $[17,18]$.

The presented genes have a direct effect on the functions of the NCC. This symporter is located at the apical membrane of the distal convoluted tubule (DCT) of the nephron. Using the sodium gradient across the apical membrane of the cells in DCT, NCC transports sodium and chloride ions from the tubular fluid into these cells. Then sodium ions are removed into the bloodstream by $\mathrm{Na}^{+}-\mathrm{K}^{+}$ATPase, while chloride ions leave the cells via the basolateral chloride channel (ClC-Kb).

The activation of NCC is directly regulated by the with-no-lysine $[\mathrm{K}]$ kinases, while KLHL3 and CUL3 are responsible for the ubiquination and further protosomeal breakdown of NCC [19].

WNK4 inhibits NCC function by reducing the amount of NCC in the cell membrane. WNK1, on the other hand, inhibits WNK4 function.

In type B PHA 2, there is a mutation in the WNK4 gene that results in the loss of its inhibitory function. In PHA 2 type $\mathrm{C}$, on the other hand, the mutation refers to the WNK1 gene and results in the increase of the WNK4 inhibition function [20]. Thus, mutations within the WNK genes result in an increased expression of the $\mathrm{Na}^{+}$$\mathrm{Cl}^{-}$co-transporter on the apical membrane of the DCT cell. This leads to an increased sodium reabsorption in the collecting duct.

$\mathrm{Na}^{+}$concentration is the main determinant of osmolarity of extracellular fluids. Changes in $\mathrm{Na}^{+}$concentration affect fluid movement and, consequently, blood volume and pressure, which is why an increase in $\mathrm{Na}^{+}$ concentration in the intravascular space will result in the development of hypertension [21]. Moreover, loss of inhibitory regulation of NCC by the WNK results in the development of hyperchloraemic metabolic acidosis, due to the increased absorption of $\mathrm{Cl}^{-}$(Fig. 2A).

The increase in sodium reabsorption by the NCC in the distal convoluted tubule reduces the activity of the epithelial sodium channel $(\mathrm{ENaC})$, which is located in the apical membrane of the epithelial cells of the collecting ducts. $\mathrm{ENaC}$ is a membrane-bound ion channel that is responsible for sodium reabsorption and is therefore the final element of natriuria regulation. In addition, it produces the necessary negative electrical gradient that drives $\mathrm{K}+$ and $\mathrm{H}+$ ion excretion.

Decreasing ENaC activity results in lowering the negative gradient in the collecting duct. Consequently, the excretion of potassium into the duct lumen by the renal outer medullary potassium channel (ROMK) is sup- pressed (Fig. 2B) [22]. Due to these electrolyte disorders, hyperkalaemia and acidosis occur in the GS.

\section{CONCLUSIONS}

In clinical practice, it is important to be vigilant for this type of hypertension, but also to be able to accurately diagnose patients who might suffer from it, because treatment often differs significantly from the treatment of idiopathic hypertension. Such an effect can significantly contribute to reducing the incidence and mortality associated with uncontrolled hypertension.

\section{DISCLOSURE}

The authors declare no conflict of interest.

\section{REFERENCES}

1. Baumgartner H, Bonhoeffer P, Westby J. ESC Guidelines for the management of grown-up congenital heart disease (new version 2010): The Task Force on the Management of Grown-up Congenital Heart Disease of the European Society of Cardiology (ESC). Eur Heart J 2010; 31: 2915-2957.

2. Paver W, Pauline G. Hypertension and hyperpotassaemia without renal disease in a young male. Med J Aust 1964; 2: 305-306.

3. Gordon RD, Geddes RA, Pawsey CG, O'Halloran MW. Hypertension and severe hyperkalaemia associated with suppression of renin and aldosterone and completely reversed by dietary sodium restriction. Australas Ann Med 1970; 19: 287-294.

4. Mayan H, Vered I, Mouallem M, et al. Pseudohypoaldosteronism type II: marked sensitivity to thiazides, hypercalciuria, normomagnesemia, and low bone mineral density. J Clin Endocrinol Metab 2002; 87: 3248-3254.

5. Gordon RD, Hodsman GP. The syndrome of hypertension and hyperkalaemia without renal failure: long term correction by thiazide diuretic. Scott Med J 1986; 31: 43-44.

6. Toka HR, Koshy JM, Hariri A. The molecular basis of blood pressure variation. Pediatr Nephrol 2013; 28: 387-399.

7. Gordon RD. Syndrome of hypertension and hyperkalemia with normal glomerular filtration rate. Hypertension 1986; 8: 93-102.

8. Mayan H, Munter G, Shaharabany M, et al. Hypercalciuria in familial hyperkalemia and hypertension accompanies hyperkalemia and precedes hypertension: description of a large family with the Q565E WNK4 mutation. J Clin Endocrin Metabol 2004; 89: 4025-4030.

9. Mayan H, Vered I, Mouallem M, et al. Pseudohypoaldosteronism type II: marked sensitivity to thiazides, hypercalciuria, normomagnesemia, and low bone mineral density. J Clin Endocrin Metab 2002; 87: 3248-3254.

10. Gamba G. Role of WNK kinases in regulating tubular salt and potassium transport and in the development of hypertension. Am J Physiol 2005; 288: F245-F252.

11. Margolis BL, Lifschitz MD. The Spitzer-Weinstein syndrome: one form of type IV renal tubular acidosis and its response to hydrochlorothiazide. Am J Kidney Dis 1986; 7: 241-244.

12. Spitzer A, Edelmann CM Jr, Goldberg LD, Henneman PH. Short stature, hyperkalemia and acidosis: a defect in renal transport of potassium. Kidney Int 1973; 3: 251-257.

13. Weinstein SF, Allan DM, Mendoza SA. Hyperkalemia, acidosis, and short stature associated with a defect in renal potassium excretion. J Pediatr 1974; 85: 355-358. 
14. Ellison DH. Pseudohypoaldosteronism Type II. 2011 Nov 10 [Updated 2017 Feb 16]. In: GeneReviews ${ }^{\circledast}$ [Internet], Adam MP, Ardinger HH, Pagon RA, et al. (eds.). University of Washington, Seattle 1993-2020.

15. Riepe FG. Clinical and molecular features of type 1 pseudohypoaldosteronism. Horm Res 2009; 72: 1-9.

16. Hadchouel J, Ellison DH, Gamba G. Regulation of renal electrolyte transport by WNK and SPAK-OSR1 kinases. Annu Rev Physiol 2016; 78: 367-389.

17. Boyden LM, Choi M, Choate KA, et al. Mutations in kelch-like 3 and cullin 3 cause hypertension and electrolyte abnormalities. Nature 2012; 482: 98-102.

18. Louis-Dit-Picard H, Barc J, Trujillano D, et al. KLHL3 mutations cause familial hyperkalemic hypertension by impairing ion transport in the distal nephron. Nat Genet 2012; 44: 456-460, S451-453.

19. Hollander R, Mortier G, van Hoeck K. Hyperkalemia in young children: blood pressure checked? Euro J Pediatr 2016; 175: 2011-2013.

20. Padmanabhan S, Caulfield M, Dominiczak AF. Genetic and molecular aspects of hypertension. Circ Res 2015; 116: 937-959.

21. O'Shaughnessy KM. Gordon Syndrome: a continuing story. Pediatr Nephrol 2015; 30: 1903-1908.

22. Vehaskari VM. Heritable forms of hypertension. Pediatr Nephrol 2009; 24: 1929-1937. 